\title{
Defining Discrete Objects for Polygonalization: The Standard Model
}

\author{
Eric Andres \\ Université de Poitiers \\ Laboratoire IRCOM-SIC, Bât. SP2MI, \\ BP 30179, 86962, Futuroscope Cedex, France
}

\begin{abstract}
A new description model, called the standard model, for discrete linear objects in dimension $\mathrm{n}$ is proposed. Standard objects are tunnel-free and (n-1)-connected. The discrete objects are defined analytically as union of intersections of half-spaces. The standard 3D polygons are well suited for polygonalization. This is the main reason why this model has been developed.
\end{abstract}

\section{Introduction}

Polygonalization of discrete objects is one of the major research problems of the discrete geometry community for many years now. The main approach used in practice is the "marching cubes" [16 type approach. In this approach, local neighbourhoods of voxels are replaced by Euclidean polygons. This means that the resulting number of polygons is proportional to the number of boundary voxels of the discrete object. As the number of boundary voxels is usually extremely important, people tend to apply simplification schemes to reduce that number of polygons and thus loose approximation quality. For a couple of years now, another approach, we call a discrete analytical polygonalization, is investigated by a number of research groups. In this approach, the aim is to decompose the boundary of discrete objects into discrete analytical polygons and then these discrete polygons into Euclidean polygons. By discrete analytical polygons we understand discrete polygons that are not defined as set of discrete points but by an analytical description that is independent of the number of discrete points composing it. The aim here is to decompose the boundary of a discrete object into a number of discrete polygons that isn't, in general, directly proportional to the number of boundary voxels. Potential applications can be found in compression, visualization, transformations, medical imaging, ...

Numerous papers have brought new insight and new ideas on how to tackle this difficult problem. However, only a small part of the problem has been solved. In this paper we propose a new element to the problem that was missing so far, the definition of a discrete analytical polygon. Several authors have proposed algorithms that determine if a given set of discrete points belong to the same Reveillès analytical discrete plane 172 . At the same time they provide the analytical description (two inequalities) of the discrete plane [91312]. Some others

A. Braquelaire, J.-O. Lachaud, and A. Vialard (Eds.): DGCI 2002, LNCS 2301, pp. 313-325 2002. (C) Springer-Verlag Berlin Heidelberg 2002 
propose a description of the equivalence class of all the possible discrete analytical planes the set of boundary points belongs to [1119. This only leads to decompositions of the boundary of discrete object into planar sections. All these different approaches couldn't go beyond a planar decomposition since nobody knew how to define discrete polygon and therefore nobody could propose a complete discrete polygonalization algorithm. Existing discrete polygons such as the ones proposed by A. Kaufman [14] are well suited for visualization purposes but can't be used for polygonalization: they are not planar (points do not all belong to a same discrete plane), not topologically consistent (sometimes with holes) and not analytically defined.

As already stated we propose in this paper a definition of a $3 \mathrm{D}$ discrete analytical polygon but also, more generally, a discrete analytical model for all linear objects in dimension $n$ (discrete points, $m$-flats and geometrical simplices). To the best authors knowledge, it is the first time that a discrete model is proposed that defines discrete objects in arbitrary dimensions. The new discrete analytical model proposed is called the standard model and is derived from the supercover model [134185]. In fact, a standard object is obtained by a rather simple rewriting process of the inequalities defining analytically a supercover object [5]. The name "standard" model derives from the "standard plane" introduced by J. Françon [10]. The standard model is called a discrete analytical model because the discrete objects (points, $m$-flats, simplices) are defined analytically by a finite number of inequalities that is independent of the set of discrete points of the object. For instance, a 3D standard triangle is defined by 17 or less inequalities independently of its size.

The model we propose has many interesting properties. It has been shown that the standard model is in fact a 0-discretisation of Brimkov, Andres and Barneva [6]7 and therefore is $(n-1)$-connected and tunnel-free. In our notation, in $3 \mathrm{D}$, our 2-connectivity corresponds to the classical 6 -connectivity. This means that our model fits particularly well polygonalization approaches such as the one proposed by L. Papier and J. Françon [12] in Khalimski-Kovalesky spaces [15, 10. The model is by definition geometrically consistent: for instance, the vertices of a 3D standard polygon are 3D standard points, the edges of a $3 \mathrm{D}$ standard polygons are $3 \mathrm{D}$ standard line segments and the $3 \mathrm{D}$ standard polygon is a piece of a $3 \mathrm{D}$ standard plane.

In section 2, we start by introducing the main notations of the paper, before briefly recalling the main properties of the supercover model. In section 3 , we introduce the orientation convention that forms the basis of the definition of the standard model before we formally define the standard model. In section 3.3, the main properties of the standard objects are presented especially the tunnelfreeness and the $(n-1)$-connectivity. In Section 3.5, we examine the different classes of standard linear objects to see how the definition is translated in practice and how the different inequalities defining the objects are established. We conclude in section 4 . 


\section{Preliminaries}

\subsection{Basic Notations}

Most of the following notations correspond to those given by Cohen and Kaufman in [8] and those given by Andres in [5]. We provide only a short recall of these notions.

Let $\mathbb{Z}^{n}$ be the subset of the $n \mathrm{D}$ Euclidean space $\mathbb{R}^{n}$ that consists of all the integer coordinate points. A discrete (resp. Euclidean) point is an element of $\mathbb{Z}^{n}$ (resp. $\mathbb{R}^{n}$ ). A discrete (resp. Euclidean) object is a set of discrete (resp. Euclidean) points. A discrete inequality is an inequality with coefficients in $\mathbb{R}$ from which we retain only the integer coordinate solutions. A discrete analytical object is a discrete object defined by a finite set of discrete inequalities. An $m$-flat is a Euclidean affine subspace of dimension $m$.

Let us consider a set $P$ of $m+1$ linearly independent Euclidean points $P^{0}, \ldots, P^{m}$. We denote $A^{m}(P)$ the $m$-flat induced by $P$ (i.e. the $m$-flat containing $P$ ). We denote $S^{m}(P)$ the geometrical simplex of dimension $m$ in $\mathbb{R}^{n}$ induced by $P$ (i.e. the convex hull of $P$ ). For $S=S^{m}(P)$ a geometrical simplex, we denote $\bar{S}=A^{m}(P)$ the corresponding $m$-flat. For a $n$-simplex $S=S^{n}(P)$, we denote $E\left(S, P^{i}\right)$ is the half-space of boundary $A^{n-1}\left(P \backslash P^{i}\right)$ that contains $P^{i}$.

We denote $p_{i}$ the $i$-th coordinate of a point or vector $p$. Two discrete points $p$ and $q$ are $k$-neighbours, with $0 \leq k \leq n$, if $\left|p_{i}-q_{i}\right| \leq 1$ for $1 \leq i \leq n$, and $k \leq n-\sum_{i=1}^{n}\left|p_{i}-q_{i}\right|$. The voxel $\mathbb{V}(p) \subset \mathbb{R}^{n}$ of a discrete $n \mathrm{D}$ point $p$ is defined by $\mathbb{V}(p)=\left[p_{1}-\frac{1}{2}, p_{1}+\frac{1}{2}\right] \times \cdots \times\left[p_{n}-\frac{1}{2}, p_{n}+\frac{1}{2}\right]$. For a discrete object $F$, $\mathbb{V}(F)=\bigcup_{p \in F} \mathbb{V}(p)$.

A $k$-path in a discrete object $A$ is a sequence of discrete points all in $A$ such that consecutive pairs of points are $k$-neighbours. A discrete object $A$ is $k$-connected if there is a $k$-path between two arbitrary points of $A$. A $k$-component is a maximal $k$-connected set. Let $D$ be a subset of a discrete object $E$. If $E \backslash D$ is not $k$-connected then $D$ is said to be $k$-separating in $E$. Let $E$ be a $k$-separating discrete object in $\mathbb{Z}^{n}$ such that $\mathbb{Z}^{n} \backslash E$ has exactly two $k$-components. A $k$-simple point in $E$ is a discrete point $p$ such that $E \backslash p$ is $k$-separating. A $k$-separating discrete object in $\mathbb{Z}^{n}$ is called $k$-minimal if it does not contain any $k$-simple points.

Let us consider two objects $F$ of dimension $n$ and $G$ of dimension $m$. The Cartesian product of $F$ and $G$ is defined by $F \times G=\{(f, g) \mid f \in F, g \in G\}$. The Minkowski sum of $F$ and $G$ is defined by $F \oplus G=\{f+g \mid f \in F, g \in G\}$. We denote $\sigma^{n}$ the set of all the permutations of $\{1, \ldots, n\}$. Let us denote $\mathbb{J}_{m}^{n}$ the set of all the strictly growing sequences of $m$ integers all between 1 and $n$ : $\mathbb{J}_{m}^{n}=\left\{j \in \mathbb{Z}^{m} \mid 1 \leq j_{1}<j_{2}<\ldots<j_{m} \leq n\right\}$. This defines a set of multi-indices. Let us consider an object $F$ in the $n$-dimensional Euclidean space $\mathbb{R}^{n}$, with $n>1$. 
The orthogonal projection is defined by:

$$
\begin{aligned}
& \pi_{i}(F)=\left\{\left(q_{1}, \ldots, q_{i-1}, q_{i+1}, \ldots, q_{n}\right) \mid q \in \mathbb{R}^{n}\right\}, \text { for } 1 \leq i \leq n ; \\
& \pi_{j}(F)=\left(\pi_{j_{1}} \circ \pi_{j_{2}} \circ \cdots \circ \pi_{j_{m}}\right)(F), \text { for } j \in \mathbb{J}_{m}^{n} .
\end{aligned}
$$

The orthogonal extrusion is defined by:

$$
\varepsilon_{j}(F)=\pi_{j}^{-1}\left(\pi_{j}(F)\right), \text { for } j \in \mathbb{J}_{m}^{n} .
$$

We define an axis arrangement application $r_{j}$, for $j \in \mathbb{J}_{m}^{n}$, by:

$$
\begin{aligned}
r_{j} & : \mathbb{R}^{n} \rightarrow \mathbb{R}^{n} \\
x & \mapsto\left(x_{\sigma_{j}(1)}, x_{\sigma_{j}(2)}, \ldots, x_{\sigma_{j}(n)}\right)
\end{aligned}
$$

where the permutation $\sigma_{j} \in \sigma^{n}$ is defined by:

$$
\sigma_{j}=\left\{\begin{array}{c}
\text { for } 1 \leq i \leq m, \sigma_{j}\left(j_{i}\right)=i \\
\text { else } \sigma_{j}\left(k_{r}\right)=i
\end{array}\right.
$$

so that $k_{r}<k_{r+1}$ and $k_{r} \neq j_{s}$ for all $1 \leq r \leq n-m$ and for all $1 \leq s \leq$ $m$. The axis arrangement application has been specifically designed so that it verifies the two following properties: $\pi_{j}(F)=\pi_{(1,2, \ldots, m)}\left(r_{j}^{-1}(F)\right)$ and $\varepsilon_{j}(F)=$ $r_{j}\left(\varepsilon_{(1,2, \ldots, m)}\left(r_{j}^{-1}(F)\right)\right)$ for all $F$ in $\mathbb{R}^{n}$ and $j \in \mathbb{J}_{m}^{n}$.

\subsection{Recalls on Supercover}

A discrete object $G$ is a cover of a Euclidean object $F$ if $F \subset \mathbb{V}(G)$ and $\forall p \in$ $G, \mathbb{V}(p) \cap F \neq \varnothing$. The supercover $\mathbb{S}(F)$ of a Euclidean object $F$ is defined by $\mathbb{S}(F)=\left\{p \in \mathbb{Z}^{n} \mid \mathbb{V}(p) \cap F \neq \varnothing\right\} . \mathbb{S}(F)$ is by definition a cover of $F$. It is easy to see that if $G$ is a cover of $F$ then $G \subset \mathbb{S}(F)$. The supercover of $F$ can be defined in different ways: $\mathbb{S}(F)=\left(F \oplus B^{\infty}\left(\frac{1}{2}\right)\right) \cap \mathbb{Z}^{n}=\left\{p \in Z^{n} \mid d^{\infty}(p, F) \leq \frac{1}{2}\right\}$ where $B^{\infty}(r)$ if the ball centered on the origin, of radius $r$ for the distance $d^{\infty}$. This links the supercover to mathematical morphology [18].

The supercover has many properties. Let us consider two Euclidean objects $F$ and $G$, and a multi-index $j \in \mathbb{J}_{m}^{n}$, then: $\mathbb{S}(F)=\bigcup_{\alpha \in F} \mathbb{S}(\alpha), \mathbb{S}(F \times G)=$ $\mathbb{S}(F) \times \mathbb{S}(G), r_{j}(\mathbb{S}(F))=\mathbb{S}\left(r_{j}(F)\right), \pi_{j}(\mathbb{S}(F))=\mathbb{S}\left(\pi_{j}(F)\right)$ and $\varepsilon_{j}(\mathbb{S}(F))=$ $\mathbb{S}\left(\varepsilon_{j}(F)\right)=r_{j}\left(\mathbb{Z}^{m} \times \mathbb{S}\left(\pi_{j}(F)\right)\right)[[]$.

Definition 1. (Bubble)

A $k$-bubble, with $1 \leq k \leq n$, is the supercover of a Euclidean point that has exactly $k$ half-integer coordinates.

A half-integer is a real $l+\frac{1}{2}$, with $l$ an integer. A $k$-bubble is formed of $2^{k}$ discrete points.

Definition 2. (Bubble-free)

The cover of an $m$-flat is said to be bubble-free if it has no $k$-bubbles for $k>m$. The cover of a simplex $S$ is said to be bubble-free if $\bar{S}$ is bubble-free. 
There are two types of bubbles in the supercover of an $m$-flat $F$. The $k$-bubbles, for $k \leq m$, are discrete points that are part of all the covers of $F$. If we remove any of these points, the discrete object isn't a cover anymore. In the $k$-bubbles, for $k>m$, there are discrete points that are "simple" points. The aim of this paper is to propose discrete analytical objects that are bubble-free by removing one of the points as illustrated by the figure.

Lemma 1. A discrete point $p$ belongs to a $k$-bubble, $k>m$, of the supercover of an $m$-flat $F$ if and only if there exists a point $\alpha \in F$ with $k$ half-integer coordinates such that $p \in \mathbb{S}(\alpha)$.

The proof of this lemma is obvious.

\section{Standard Model}

The aim of this paper is to propose a new cover class, called the standard cover. This cover is so far only defined for linear objects in all dimensions. This discrete analytical model has been designed to conserve most of the properties of the supercover, to be bubble-free and $(n-1)$-connected.

\subsection{Orientation Convention}

The standard model, contrary to the supercover, is not unique. It depends on the choice of an orientation convention. We need one orientation convention per dimension $\mathbb{R}^{m}, m>0$. This choice must then remain unchanged for all the primitives handled. The choice of an orientation convention per dimension has to be coherent with the operator $\pi$. This means that we want the following property to be verified: $\mathbb{S} t\left(\pi_{j}(F)\right)=\pi_{j}(\mathbb{S} t(F))$. If this is not the case, we won't have correct modeling properties. In general, with arbitrary orientation conventions there is no reason for this property to be verified. It can sometimes be tricky to find a "good" set of orientation conventions. We propose a set of orientation conventions, denoted $O^{n}$ and called the basic orientation conventions, that verify the above mentioned property.

Definition 3. (Standard orientation)

Let us consider a discrete analytical half-space $E: \sum_{i=1}^{n} C_{i} X_{i} \leq B$ and the basic orientation convention $O^{n}$. We say that $E$ has a standard orientation if :

$-C_{1}>0$

- or if $C_{1}=0$ and $C_{2}>0$;

- :

- or if $C_{1}=\cdots=C_{n-1}=0$ and $C_{n}>0$.

If $E$ has not a standard orientation then we say that $E$ has a supercover orientation. 
We consider from now on, without loss of generality, only the basic orientation conventions for all $n>0$. All the standard primitives are defined with these basic orientation conventions. The basic orientation conventions are coherent with respect to the operators $\pi$. After $\pi_{j}$, for $j \in \mathbb{J}_{m}^{n}$, the orientation convention $O^{n}$ in $\mathbb{R}^{n}$ becomes $O^{n-m}$ in $\mathbb{R}^{n-m}$.

\subsection{Standard Model Definition}

We now have gathered all the elements we need to define the standard discretisation model of linear objects in $\mathbb{R}^{n}$.

Definition 4. (Standard Model)

Let $F$ be a linear Euclidean object in $\mathbb{R}^{n}$ whose supercover is described analytically by a finite set of inequalities $F_{k}: \sum_{i=1}^{n} C_{i, k} X_{i} \leq B_{k}$. The standard model $\mathbb{S t}(F)$ of $F$, for the basic orientation convention $O^{n}$, is the discrete object described analytically by a finite set of discrete inequalities $F_{k}^{\prime}$ obtained by substituting each inequality $F_{k}$ by $F_{k}^{\prime}$ defined as follows:

- If $F_{k}$ has a standard orientation then $F_{k}^{\prime}: \sum_{i=1}^{n} C_{i, k} X_{i}<B_{k}$;

- else $F_{k}^{\prime}: \sum_{i=1}^{n} C_{i, k} X_{i} \leq B_{k}$.

This definition is algorithmically easy to set up. Once a discrete analytical description of an object is available, the transition from the supercover model to the standard model and vice-versa is trivial.

\subsection{Properties}

We are now going to present the most important properties of standard objects. Let us consider a Euclidean linear object $F$ of topological dimension $m$ in $\mathbb{R}^{n}$. We have by definition $\mathbb{S} t(F) \subset \mathbb{S}(F)$ even more precisely, if $p \in \mathbb{S}(F) \backslash \mathbb{S} t(F)$, then $d^{\infty}(p, F)=\frac{1}{2}$. A standard object is a supercover object from which we have removed some discrete points. These points are all at a distance $\frac{1}{2}$ from the Euclidean primitive. We have $\mathbb{S} t(F)=\mathbb{S}(F)$ if no point with at least $m+1$ half-integer coordinates belongs to the boundary of $F$. The differences between the supercover of $F$ and the standard model of $F$ are located in the $k$-bubbles of $F$, for $k>m$. One of the immediate consequences of this is that the standard model remains a cover: $F \subset \mathbb{V}(\mathbb{S} t(F))$. It is because of this property that the standard model is also sometimes called standard cover.

The standard model retains most of the set properties of the supercover. It is easy to deduce from definition 4 , that if we have two Euclidean linear objects $F$ and $G$ in $\mathbb{R}^{n}$, then:

$$
\begin{aligned}
& \mathbb{S} t(F \cup G)=\mathbb{S} t(F) \cup \mathbb{S} t(G) ; \mathbb{S} t(F \cap G) \subset \mathbb{S} t(F) \cap \mathbb{S} t(G) \\
& F \subset G \Rightarrow \mathbb{S t}(F) \subset \mathbb{S t}(G) ; \quad \mathbb{S t}(F \times G)=\mathbb{S t}(F) \times \mathbb{S t}(G) ; \\
& \mathbb{S} t\left(\pi_{j}(F)\right)=\pi_{j}(\mathbb{S} t(F)) ; \quad \mathbb{S} t\left(\varepsilon_{j}(F)\right)=\varepsilon_{j}(\mathbb{S} t(F)) .
\end{aligned}
$$


The first property ensures that we'll be able to construct complex discrete objects out of basic elements such as simplices. These last properties are characteristic of correct orientation conventions. The properties are only verified if the orientation conventions are defined for all dimensions lower or equal to $n$ and if they are coherent with respect to the operator $\pi$. This is the case for the basic orientation conventions $O^{k}$, for $k \leq n$.

It is important to notice that, in general, $\mathbb{S} t(F) \neq \bigcup_{\alpha \in F} \mathbb{S t}(\alpha)$. This property of the supercover is not conserved. We have $\mathbb{S} t(F \cup G)=\mathbb{S t}(F) \cup \mathbb{S} t(G)$ for a union of a finite number of objects. This comes simply from the fact that the standard model is not defined for an analytical description that has an infinite number of discrete inequalities. One simple example for that is given by the $2 \mathrm{D}$ line $D: x_{1}-x_{2}=0$. The standard model of the line is $\mathbb{S} t(D):-1 \leq x_{1}-x_{2}<1$ while $\bigcup_{\alpha \in F} \mathbb{S t}(\alpha)=\left\{x \in \mathbb{Z}^{2} \mid x_{1}-x_{2}=0\right\}$.

One of the main properties of the standard model concerns the connectivity and the tunnel-freeness:

Theorem 1. (connectivity and tunnel-freeness)

Let $F$ be a Euclidean linear object of topological dimension $m$ in $\mathbb{R}^{n}$. Its standard model $\mathbb{S} t(F)$ is $(n-1)$-connected and tunnel-free.

The standard model is a particular case of $k$-discretisations as introduced by Brimkov, Andres and Barneva in [67]. It is shown that the standard model is in fact a 0 -discretisation (Theorem 3 in [7]) and that 0-discretisations are $(n-1)$ connected and tunnel-free (proposition 3 in [6] and theorem 4 in [7]). Another property proved in [18,7] is that the standard model minimizes the Hausdorff distance with the Euclidean object. See [18,6]7] for details.

\subsection{Description of Standard Primitives}

We'll examine now the discrete analytical description of the different classes of standard linear primitives (half-space, point, $m$-flat and $m$-simplex) and how they can be computed. Our purpose here is to propose a discretisation scheme that can be used is practical applications. By definition 4, every analytical description of a standard linear primitive is based on the analytical description of a standard half-space. That is the one we present first. We deduce from it the formulas for the standard point, $m$-flat and $m$-simplex in the sections that follow.

Standard Half-space. The standard half-space is given by :

Proposition 1. (Standard half-space)

Let us consider a Euclidean half-space $E: \sum_{i=1}^{n} C_{i} X_{i} \leq B$. The standard model $\mathbb{S} t(E)$ of $E$, according to an orientation convention, is analytically described by: 
- If E has a standard orientation then

$$
\mathbb{S} t(E)=\left\{p \in \mathbb{Z}^{n} \mid \sum_{i=1}^{n} C_{i} p_{i}<B+\frac{\sum_{i=1}^{n}\left|C_{i}\right|}{2}\right\} ;
$$

- else

$$
\mathbb{S} t(E)=\left\{p \in \mathbb{Z}^{n} \mid \sum_{i=1}^{n} C_{i} p_{i} \leq B+\frac{\sum_{i=1}^{n}\left|C_{i}\right|}{2}\right\} .
$$

The proposition is an immediate extension to dimension $n$ of results on the supercover [1345] and of definition 4.

Standard point. The analytical description of a standard point can easily be deduced from the one of the standard half-space. It is however interesting to notice that the standard discretisation of a Euclidean point is always composed of one and only one discrete point contrary to what happens with a supercover discretization of a Euclidean point that can be formed of $2^{k}$ points, $0 \leq k \leq n$.

Proposition 2. (Standard point)

Let us consider a Euclidean point $\alpha \in \mathbb{R}^{n}$ and the basic orientation convention $O^{n}$. The standard model $\mathbb{S} t(\alpha)$ of $\alpha$ is the discrete point:

$$
\mathbb{S} t(\alpha)=\left(\left\lceil\alpha_{1}-\frac{1}{2}\right\rceil, \ldots,\left\lceil\alpha_{n}-\frac{1}{2}\right\rceil\right)
$$

The proof is obvious. In figure1 the cross represents the Euclidean point. The black dot represents the corresponding discrete standard point. The square with the doted lines represent the zone covered by the 4 inequalities corresponding to the analytical description of a standard point.

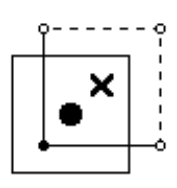

(a)

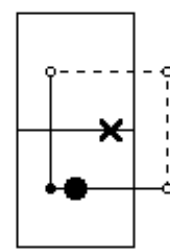

(b)

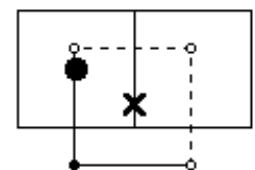

(c)

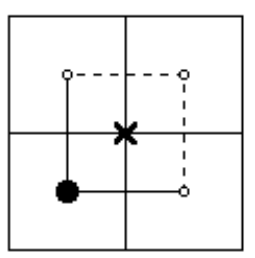

(d)

Fig. 1. Different configurations of 2D standard points. 
Standard $\boldsymbol{m}$-flat. One of the consequences of the properties $\mathbb{S} t\left(\pi_{j}(F)\right)=$ $\pi_{j}(\mathbb{S} t(F))$ and $\mathbb{S} t\left(\varepsilon_{j}(F)\right)=\varepsilon_{j}(\mathbb{S} t(F))$ is that the formulas that lead to the discrete analytical description of a standard $m$-flat or standard $m$-simplex are simple transpositions of the formulas that have been established for the supercover [5].

Proposition 3. (Standard $m$-flat)

Let us consider an $m$-flat $F$ in $\mathbb{R}^{n}$ and the basic orientation conventions $O^{k}$, for all $k>0$.

a) If $F$ is a 0 -flat in $\mathbb{R}^{n}$, we apply proposition $\mathbf{2}$,

b) If $F$ is a $(n-1)$-flat, we apply twice proposition 1

c) else the analytical description of the standard model of $F$ is given by:

$$
\mathbb{S} t(F)=\bigcap_{j \in \mathbb{J}_{n-1-m}^{n}} \mathbb{S} t\left(\varepsilon_{j}(F)\right)=\bigcap_{j \in \mathbb{J}_{n-1-m}^{n}} r_{j}\left(\mathbb{Z}^{m} \times \mathbb{S} t\left(\pi_{j}(F)\right)\right)
$$

We reapply then, recursively, proposition [3 on $\mathbb{S} t\left(\pi_{j}(F)\right)$ for all $j \in \mathbb{J}_{n-1-m}^{n}$.

This proposition is composed of several steps corresponding to the algorithm that yields the analytical description of the standard model of an $m$-flat. Let us discuss step c). The formula $\mathbb{S} t(F)=\bigcap_{j \in \mathbb{J}_{n-1-m}^{n}} \mathbb{S} t\left(\varepsilon_{j}(F)\right)$ alone is not sufficient to describe the standard $m$-flat, with $0<m<n-1$, since $\varepsilon_{j}(F)$ is not necessarily a hyperplane in $\mathbb{R}^{n}$. We might even have $F=\varepsilon_{j}(F)$ for some $j \in \mathbb{J}_{n-1-m}^{n}$. The way around this problem is to examine $\pi_{j}(F)$ in $\mathbb{R}^{m+1}$. The new orientation convention for $\mathbb{R}^{m+1}$ after $\pi_{j}$ is $O^{m+1}$.

We have different cases that occur:

- If $\pi_{j}(F)$ is a hyperplane in $\mathbb{R}^{m+1}$ then $\varepsilon_{j}(F)$ is a hyperplane in $\mathbb{R}^{n}$. We do not actually need to consider $\pi_{j}(F)$. We could directly use case b) on $\mathbb{S t}\left(\varepsilon_{j}(F)\right)$.

- If $\pi_{j}(F)$ is a point in $\mathbb{R}^{m+1}$ then we consider case a) in $\mathbb{R}^{m+1}$, with the basic orientation convention, and formula $r_{j}\left(\mathbb{Z}^{m} \times \mathbb{S} t\left(\pi_{j}(F)\right)\right)$.

- If $\pi_{j}(F)$ is a $k$-flat, $0<k<m$, in $\mathbb{R}^{m+1}$ then we consider again case c), with the basic orientation convention. We have, by definition, $\mathbb{S} t\left(\pi_{j}(F)\right)=$ $\bigcap \mathbb{S} t\left(\varepsilon_{j^{\prime}}\left(\pi_{j}(F)\right)\right)$. We repeat the operation described in case c) for $j^{\prime} \in \mathbb{J}_{m-k}^{m+1}$

$\pi_{j^{\prime}}\left(\pi_{j}(F)\right)$ in $\mathbb{R}^{k+1}$.

We know that this process ends since each time we repeat case c) we consider a new object in a space of strictly lower dimension.

Standard Simplex. Let us finish with the formulas describing a standard simplex. These formulas are a direct transposition of the formulas obtained for the supercover [5]. 

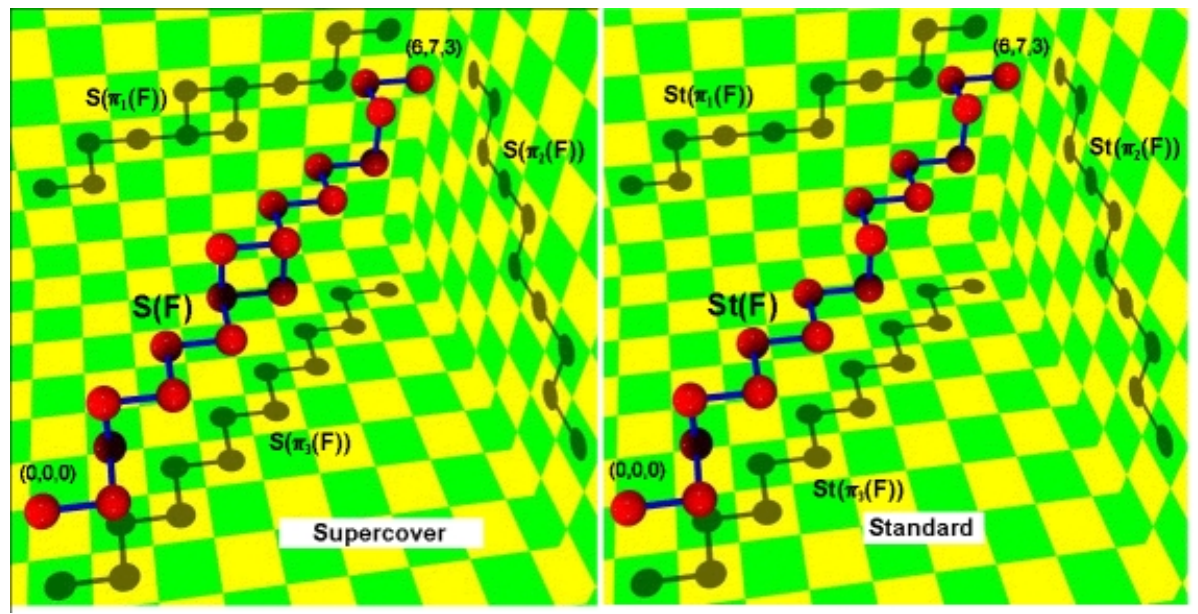

Fig. 2. Supercover and Standard 3D line.

Proposition 4. (Standard Simplex)

Let us consider a simplex $S=\left\{P^{0}, \ldots, P^{m}\right\}$ of dimension $m$ and the basic orientation conventions $O^{k}$, for all $k>0$. The standard model of $S$ is defined by:

a) If $m=n$ then $\mathbb{S t}(S)=\left(\bigcap_{i=0}^{n} \mathbb{S} t\left(E\left(S, P^{i}\right)\right)\right) \cap\left(\bigcap_{j=1}^{n} \mathbb{S} t\left(\varepsilon_{j}(S)\right)\right)$;

b) If $m=n-1$ then $\mathbb{S} t(S)=\mathbb{S} t(\bar{S}) \cap\left(\bigcap_{j=1}^{n} \mathbb{S} t\left(\varepsilon_{j}(S)\right)\right)$;

c) If $m \leq n-2$ then $\mathbb{S t}(S)=\bigcap_{j \in \mathbb{J}_{n-m-1}^{n}} \mathbb{S} t\left(\varepsilon_{j}(S)\right)$.

Let us just recall some notations. If $S$ is a simplex of dimension $m$ then we denote $\bar{S}$ the $m$-flat containing all the points defining $S$. If $S$ is a simplex of dimension $n$ in $\mathbb{R}^{n}$ then we denote $E\left(S, P^{i}\right)$ the half-space that contains $P^{i}$ and of boundary the $(n-1)$-flat $\overline{\left\{P^{0}, \ldots, P^{i-1}, P^{i+1}, \ldots, P^{m}\right\}}$. Figure 3 shows three views of a standard 3D triangle: Figure 3(a) presents a classical, voxel view, of the standard triangle; figure 3 (b) presents the same triangle in a $\mathrm{K}^{2}$-space representation; finally figure 3(c) represents what we have called the analytical view and represents the 17 inequalities describing the standard 3D triangle.

\section{Conclusion}

We have defined in this paper the standard model for half-spaces, $m$-flats and $m$-simplices in dimension $n$. This is, to the authors best knowledge, one of the first times that discrete primitives are described analytically in dimension $n$. The standard model is geometrically consistent, defined analytically and standard objects are tunnel-free and $(n-1)$-connected. It seems to us that the path 
towards discrete polygonalization will be much easier with standard polygons than with other notions and this for several reasons: the standard objects are all topologically consistent. This is not the case for the discrete naïve model for instance. Most planar recognition algorithms have been designed to recognize discrete naïve plane pieces 919 . However, it is not very difficult to show that if the model is geometrical consistent then $3 \mathrm{D}$ discrete naive edges of polygons aren't connected in general [5] and 3D nä̈ve vertices might be composed of zero discrete points. This will make it very difficult to perform any polygon reconstruction process. Designing a polygon reconstruction algorithm is the next step towards polygonalization. Indeed, the following steps need to be accomplished in order to perform a discrete polygonalization: first we need to decompose the boundary of the discrete object into discrete plane pieces. This part, as we have recalled in the introduction, has been realised by several different approaches in the past. Secondly, one needs to describe the plane pieces as discrete polygons. This supposes that we know what a discrete polygon is (our paper) and that we perform some edge and vertex recognition algorithm. This is still an open and somehow difficult question.

In order to facilitate the implementation and the test of such polygonalization algorithms, we are developing a discrete modeling tool, called SpaMod (for Spatial Modeler), at the University of Poitiers (France). The standard model, as well as the supercover model, are part of the discrete objects models handled by Spamod. Spamod is still in the preliminary stages of its development, however the images of Figure 3 have been produced with this software.

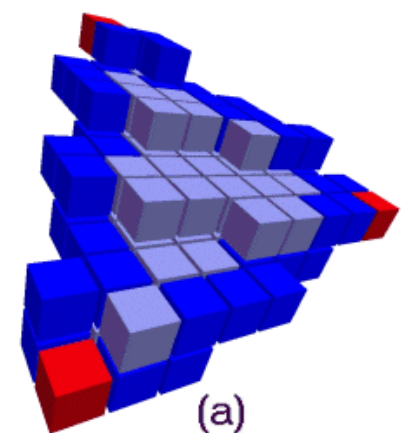

(a)

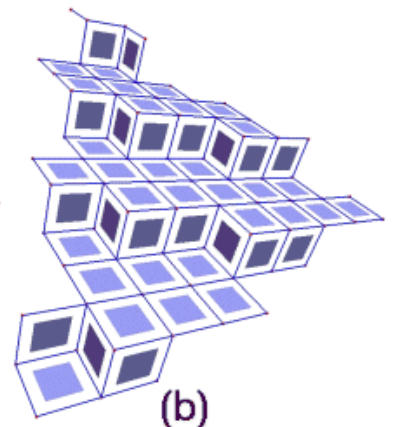

(b)

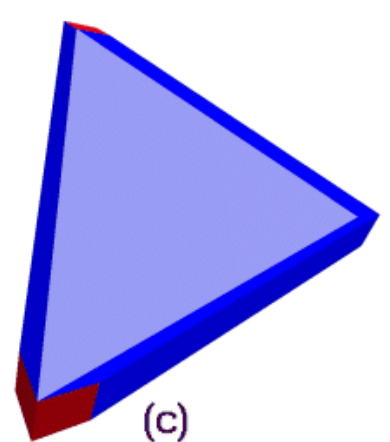

(c)

Fig. 3. Standard triangle with (a) Voxel view (b) $\mathrm{K}^{2}$-space view (c) Analytical view

Acknowledgments. The images of Figure 3 have been produced in Spamod with help of the algorithms developed by Martine Dexet. 


\section{References}

1. E. Andres, C. Sibata and R. Acharya, Supercover 3D Polygon, in: Proc. $6^{\text {th }}$ Int. Workshop on Discrete Geometry for Computer Imagery, Lyon (France), Lecture Notes in Computer Science, vol. 1176 (Springer, Berlin-Heidelberg, 1996) 237-242.

2. E. Andres, R. Acharya and C. Sibata, Discrete Analytical Hyperplanes, Graphical Models and Image Processing 59-5 (1997) 302-309.

3. E. Andres, Ph. Nehlig and J.Françon, Tunnel-free supercover 3D polygons and polyhedra, in: Proc. Eurographics '97, Budapest (Hungary), Computer Graphics Forum 16-3 (1997) C3-C13.

4. E. Andres, Ph. Nehlig and J. Françon, Supercover of Straight Lines, Planes and Triangles, in: Proc. $7^{\text {th }}$ Int. Workshop on Discrete Geometry for Computer Imagery, Montpellier (France), Lecture Notes in Computer Science, vol. 1347 (Springer, Berlin-Heidelberg, 1997) pp. 243-253.

5. E. Andres, Modélisation analytique discrète d'objets géométriques, Habilitation (in french), Laboratoire IRCOM-SIC, University of Poitiers (France), 8 Dec. 2000.

6. V.E. Brimkov, E. Andres, R.P. Barneva, Object discretizations in high dimensions and membership recognition, Proc. $9^{\text {th }}$ Int. Workshop on Discrete Geometry for Computer Imagery, Uppsala (Sweden), Lecture Notes in Computer Science, vol. 1953 (Springer, Berlin-Heidelberg, 2000) pp. 210-221..

7. V.E. Brimkov, E. Andres, R.P. Barneva, Object discretizations in high dimensions, accepted for publication in Pattern Recognition Letters.

8. D. Cohen and A. Kaufman, Fundamentals of Surface Voxelization, Graphical Models and Image Processing 57-6 (1995).

9. I. Debled-Renesson, J-P. Reveillès, A linear algorithm for digital plane recognition, 4th int. workshop in discrete geometry for computer imagery, Grenoble (France), sept. 1994.

10. J. Françon, Discrete Combinatorial Surfaces, Graphical Models and Image Processing, vol. 57, Janv. 1995.

11. J. Françon, J-M. Schramm, M. Tajine, Recognizing arithmetic straight lines and plane, 6th int. workshop in discrete geometry for computer imagery, Lyon (France), LNCS n 1176 , nov. 1996, pp. 141-150.

12. J. Françon, L. Papier, Polyhedrization of the boundary of a voxel object, in: Proc. $8^{\text {th }}$ Int. Workshop on Discrete Geometry for Computer Imagery, Marne-laVallée (France), Lecture Notes in Computer Science, vol. 1568 (Springer, BerlinHeidelberg, 1999) 425-434.

13. Y. Gérard, Local configurations of digital hyperplanes, 8th int. Workshop on discrete geometry for computer imagery, Marne-la-Vallée (France), LNCS n ${ }^{\circ} 1568$, mars 1999, pp. 365-374.

14. A. Kaufman, An algorithm for 3D scan conversion of polygons, in: Proc. of Eurographics '87, Amsterdam, (1987).

15. V. Kovalesky, Digital geometry based on the topology of abstract cell complexes, 3rd Discrete Geometry Conference in Imagery, Strasbourg (France), 1993, pp. 259284.

16. W. Lorensen, H. Cline, Marching Cubes: a high resolution 3d surface construction algorithm. SIGGRAPH'87, Anaheim (USA). Computer Graphics J., vol. 21, n 4 , Jul. 1987, pp. 163-169.

17. J-P. Réveilles, Géométrie Discrète, calculs en nombres entiers et algorithmique, State Thesis (in french), Département d'Informatique, Université Louis Pasteur, Strasbourg (France), 1991. 
18. M. Tajine, D. Wagner, C. Ronse, Hausdorff discretizations and its comparison to other discretization schemes, in: Proc. $8^{\text {th }}$ Int. Workshop on Discrete Geometry for Computer Imagery, Marne-la-Vallée (France), Lecture Notes in Computer Science, vol. 1568 (Springer, Berlin-Heidelberg, 1999) 399-410.

19. J. Vittone, J-M. Chassery, $(n-m)$-cubes and Farey nets for naïve planes understanding, 8th int. workshop in discrete geometry for computer imagery, Marne-laVallée (France), LNCS n 1568 , mars 1999, pp. 76-87. 